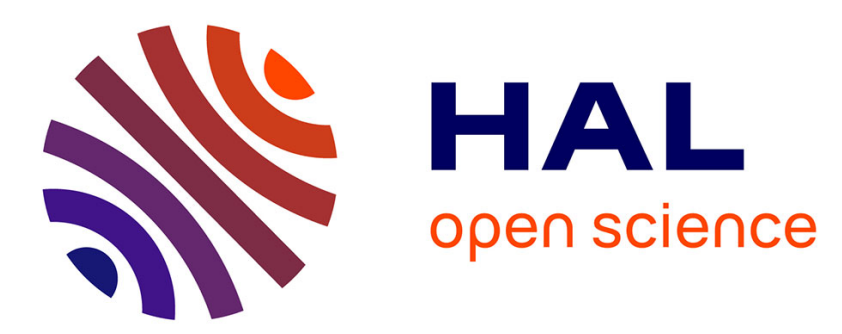

\title{
High-amplitude sound propagation in acoustic transmission-line metamaterial
}

Jiangyi Zhang, Vicente Romero-García, Georgios Theocharis, Olivier Richoux, V. Achilleos, Dimitrios Frantzeskakis

\section{- To cite this version:}

Jiangyi Zhang, Vicente Romero-García, Georgios Theocharis, Olivier Richoux, V. Achilleos, et al.. High-amplitude sound propagation in acoustic transmission-line metamaterial. Applied Physics Letters, 2021, 118 (10), pp.104102. 10.1063/5.0040702 . hal-03436122

\section{HAL Id: hal-03436122 \\ https://hal.science/hal-03436122}

Submitted on 22 Nov 2021

HAL is a multi-disciplinary open access archive for the deposit and dissemination of scientific research documents, whether they are published or not. The documents may come from teaching and research institutions in France or abroad, or from public or private research centers.
L'archive ouverte pluridisciplinaire HAL, est destinée au dépôt et à la diffusion de documents scientifiques de niveau recherche, publiés ou non, émanant des établissements d'enseignement et de recherche français ou étrangers, des laboratoires publics ou privés. 


\title{
High-amplitude sound propagation in acoustic transmission-line metamaterial
}

\author{
Jiangyi Zhang, ${ }^{1}, 2$,a) Vicente Romero-García, ${ }^{1}$ Georgios Theocharis, ${ }^{1}$ Olivier Richoux,${ }^{1}$ Vassos Achilleos, ${ }^{1}$ and \\ Dimitrios J. Frantzeskakis ${ }^{3}$ \\ ${ }^{1)}$ Laboratoire d'Acoustique de l'Université du Mans (LAUM), UMR CNRS 6613, \\ Institut d'Acoustique - Graduate School (IA-GS), CNRS, Le Mans Université, \\ France \\ ${ }^{2)}$ Faculty of Civil Engineering and Geosciences, Delft University of Technology, Delft, \\ Netherlands \\ ${ }^{3)}$ Department of Physics, National and Kapodistrian University of Athens, 15784 Athens, \\ Greece
}

(Dated: 22 November 2021)

We report experiments on high-amplitude sound wave propagation in an acoustic metamaterial composed of an air-filled waveguide periodically side-loaded by holes. In addition to the linear visco-thermal and radiation losses, high amplitude sound waves at the locations of the side holes introduce nonlinear losses. The latter, result in an amplitude-dependent reflection, transmission and absorption, which we experimentally characterize. First, we evidence that nonlinear losses change the nature of the device from a reflective to an absorbing one, showing the possibility to use the system as a nonlinear absorber. Second, we study the second-harmonic generation and its beating phenomenon both experimentally and analytically. We find that when considering the propagation of both the fundamental and the second harmonic, nonlinear losses cannot be neglected. Our results reveal the role of nonlinear losses in the proposed device, and also provide a quite accurate analytical model to capture the effect of such losses.

Acoustic metamaterials is undoubtedly an important research theme in recent years. A plethora of applications have been developed, including acoustic cloaking ${ }^{1-4}$, perfect absorbers ${ }^{5,6}$, sound focusing based on gradient index lenses $^{7-9}$, acoustic topological systems ${ }^{10-12}$, and others. In addition, recently, nonlinear acoustic metamaterials have been receiving increased attention through studies, e.g., on acoustic diode ${ }^{13,14}$, bifurcation-based acoustic switching and rectification ${ }^{15}$, nonlinear acoustic lenses ${ }^{16}$, and acoustic solitons ${ }^{17}$. However, so far, only few works have systematically considered the effect of dissipation in nonlinear acoustic metamaterials, although losses are non-negligible and should not be ignored. For example, Henríquez et al. ${ }^{18}$ found that losses could destroy the predicted behaviour of double negative acoustic metamaterials designed by Gracía-Salgado et al. ${ }^{19}$. In some works, weak dissipation was associated with linear losses ${ }^{20-22}$; however, in acoustics, nonlinear losses may easily appear, e.g., due to geometrical discontinuities ${ }^{23,24}$.

In this work, we experimentally and analytically study high-amplitude waves propagating in an acoustic metamaterial. In particular, we consider an air-filled waveguide periodically side-loaded by holes with sharp edges (geometrical discontinuities). This structure exhibits both linear and nonlinear losses. The former, are due to the radiation and viscothermal boundary layers, while the later appear due to jet and vortices formation at the locations of holes, when the wave amplitude is sufficiently high. We characterize the nonlinear losses by performing amplitude-dependent experiments measuring the reflection, transmission and absorption coefficients. First

\footnotetext{
a) Electronic mail: zhangjiangyi0607@gmail.com
}

evidence for nonlinear losses is that they result in the transformation of this metamaterial from a sound reflective system to a sound absorptive one. Pertinent results suggest the possibility of designing a perfect absorber, which is also a timely research topic in acoustics ${ }^{5,6}$. Second evidence for the nonlinear behaviour of the system is the generation of higher harmonics. In that regard, it is mentioned that Lombard et al. ${ }^{25}$ studied acoustic second-harmonic generation by a single layer of bubbles, while Bradley ${ }^{20}$ and $\mathrm{Li}$ et al. ${ }^{26}$ showed the beating of the second harmonic in acoustic metamaterial periodically loaded with resonators or scatterers. However, in these works, the effect of nonlinear losses was not highlighted. Here, we experimentally study second-harmonic generation, and measure its beating due to dispersion in the presence of nonlinear losses. We provide an analytical model to capture the effect of the nonlinear losses in both the scattering coefficients and in the higher harmonics. Our analytical results clearly illustrate that nonlinear losses play an important role in the structure.

The experimental set-up used for the characterization of the amplitude-dependent reflection, transmission and absorption is shown in Fig. 1 (a). It consists of a $3 \mathrm{~m}$ air-filled acoustic cylindrical waveguide with internal radius $r=0.025 \mathrm{~m}$, periodically loaded by 40 side-holes with a periodicity of $d=0.05 \mathrm{~m}$. At one of the ends of the waveguide, there is a source radiating white noise with frequencies from $200 \mathrm{~Hz}$ to $1000 \mathrm{~Hz}$. The other end is closed with a properly designed anechoic termination to avoid back reflections, in the frequency range of $10-1000 \mathrm{~Hz}^{27,28}$. The radius of the side holes is $r_{H}=0.0035 \mathrm{~m}$, while its length is $l_{H}=0.005 \mathrm{~m}$, equal to the wall thickness of the waveguide. Because the structure is open to the environment through the holes, the experiments take place in an anechoic room. 

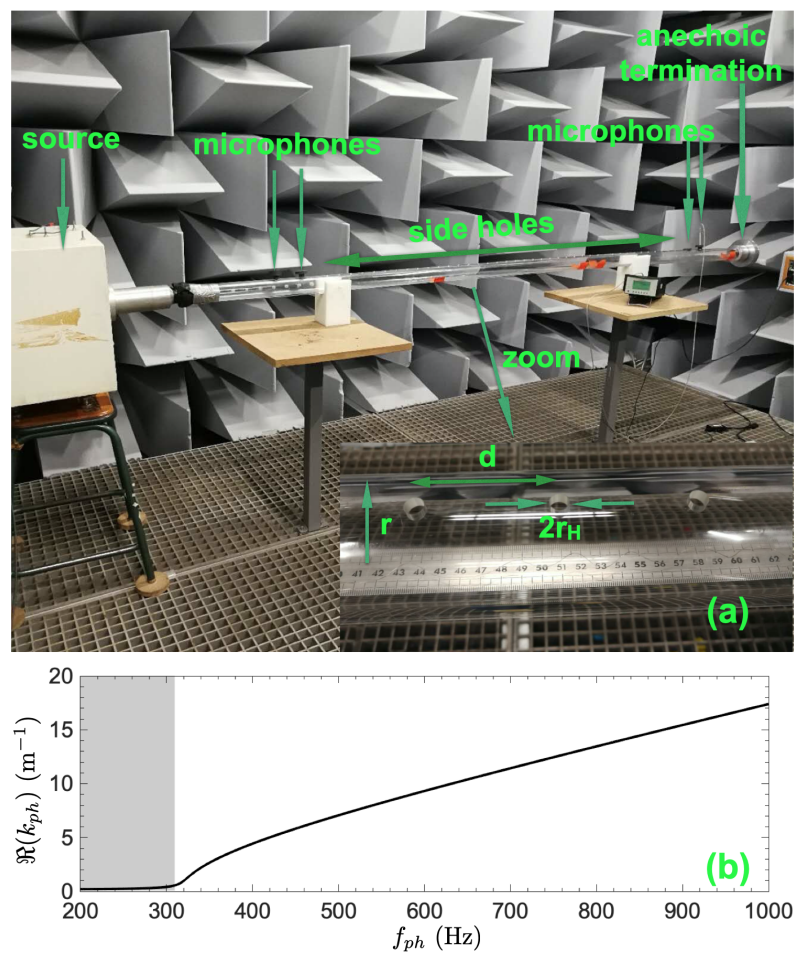

FIG. 1. (color online) (a) Picture of the experimental setting for the measurement of the amplitude-dependent reflection, transmission and absorption coefficients. (b) Dispersion relation of the system without considering nonlinear losses; the shaded area stands for the band gap.

We perform four experiments with different source amplitudes, and use a four microphone method ${ }^{29}$ to experimentally determine the amplitude-dependent transmission, reflection and absorption for each case. A pair of microphones $(1 / 2$ inch $B \& K)$ connected to each side of the structure is employed, allowing the measurement of both the forward and backward waves for the fundamental frequency. Here, we only consider the fundamental frequency, and neglect the second harmonic (as we will show later, the amplitude of the second harmonic is $40 \mathrm{~dB}$ lower than the fundamental frequency amplitude).

We start by using a small-amplitude signal around $100 \mathrm{~dB}$ (black circles in Fig. 2), which can be considered as the linear limit. As shown in Ref. [30], in this case the system features a band gap from zero- up to a cut-off-frequency, marked by the gray area in Fig. 2, and also shown in the real part of the dispersion relation in Fig. 1 (b). Then, we increase the excitation amplitude to achieve pressure amplitudes of $120 \mathrm{~dB}$ (green squares in Fig. 2), $130 \mathrm{~dB}$ (blue stars in Fig. 2) and $140 \mathrm{~dB}$ (red triangles in Fig. 2). The amplitude level is determined at a reference frequency chosen to be $600 \mathrm{~Hz}$. The experimental results are shown with the markers in Figs. 2 (a), (b) and (c), where the amplitudes of the coefficients $R$ (reflection), $T$ (transmission) and $\alpha$ (absorption) are respectively plotted as functions of frequency for each amplitude. When the amplitude of the source is increased, both the reflection and the transmission coefficients decrease. This leads to a significant enhancement of the absorption coefficient (maximum value) and a broadening of its bandwidth. According to our analytical findings, this enhanced absorption is due to the nonlinear losses appearing at the side holes. In addition, we note that in the low frequency regime (around $200 \mathrm{~Hz}$, namely in the band gap of the structure), the absorption is significantly enlarged ( 8 times larger) transforming the meta-structure from a sound reflective to a sound absorptive one.

This amplitude-dependent phenomenon that we observe is also well captured by the transfer matrix method, using a nonlinear impedance model for the side-holes ${ }^{23,24}$ (see solid lines in Fig. 2). According to this approach (see details in the Supplementary Material), the $n$-th side hole is characterized by a lumped element of impedance $Z_{H(n)}=Z_{L}+Z_{N L(n)}$, composed by $(i)$ a linear part, due to both hole geometry and the radiation losses, and (ii) a nonlinear impedance (resistance), given by:

$$
Z_{N L(n)}=\beta_{H} Z_{c H} M_{(n)} S t_{(n)}^{1 / 3},
$$

where $Z_{c H}$ is the characteristic impedance of a hole ${ }^{31}$, $M_{(n)}$ is the acoustic Mach number, $S t_{(n)}$ is the acoustic Strouhal number ${ }^{23,24,32}$, and $\beta_{H}$ is a "fitting" parameter. In Refs. [33] and [34], $\beta_{H}$ is determined by means of numerical simulations: Disselhorst and Wijngaarden ${ }^{33}$ found values of $\beta_{H}$ between 0.6 and 1.0, while Peters and Hirschberg ${ }^{34}$ evaluate $\beta_{H}$ to 0.2 . In our case, by direct comparison with experiments, we found that $\beta_{H}$ depends on the amplitude as $\beta_{H(140 \mathrm{~dB})}=0.6, \beta_{H(130 \mathrm{~dB})}=0.4$, $\beta_{H(120 \mathrm{~dB})}=0.3$, while $\beta_{H(100 \mathrm{~dB})}=0$ (linear case), i.e., the side holes feature no nonlinear losses when the amplitude of the source is small $(\lesssim 100 \mathrm{~dB})$.

We now proceed with experiments regarding the second harmonic generation. The set-up is similar to that of the previous experiment, except that the number of holes is increased to 60 , so as to better capture the beating phenomenon of the second harmonic. The signal source is now sinusoidal, with a fixed frequency $f_{p h}=600 \mathrm{~Hz}$. This driving frequency is chosen inside the propagating band, but close enough to the cutoff frequency, where the effects of dispersion and nonlinearity are stronger.

Employing the transmission line (TL) approach developed in Ref. [21], we have analytically derived the pressure distribution of the fundamental, $p_{\omega_{p h}}(x)$, and the second harmonic, $p_{2 \omega_{p h}}(x)$ (see Suplemental material for details). Pertinent analytical expressions are obtained using the continuum approximation, leading to a nonlinear partial differential equation for the pressure, which is analyzed by means of perturbation theory, and upon considering an input signal that fits the experimental source. The source generates a weak second harmonic component, defined as $\left.p_{2 \omega_{p h}}\right|_{(x=0)}=A_{a}^{p h} \cos \left(2 \omega_{p h} t\right)$ where $A_{a}^{p h}$ is measured at the vicinity of the source, and is found to be $40 \mathrm{~dB}$ less than the amplitude of the fundamental component. Therefore, in the analytical expression of $p_{2 \omega_{p h}}$, we have to incorporate an additional term due to 

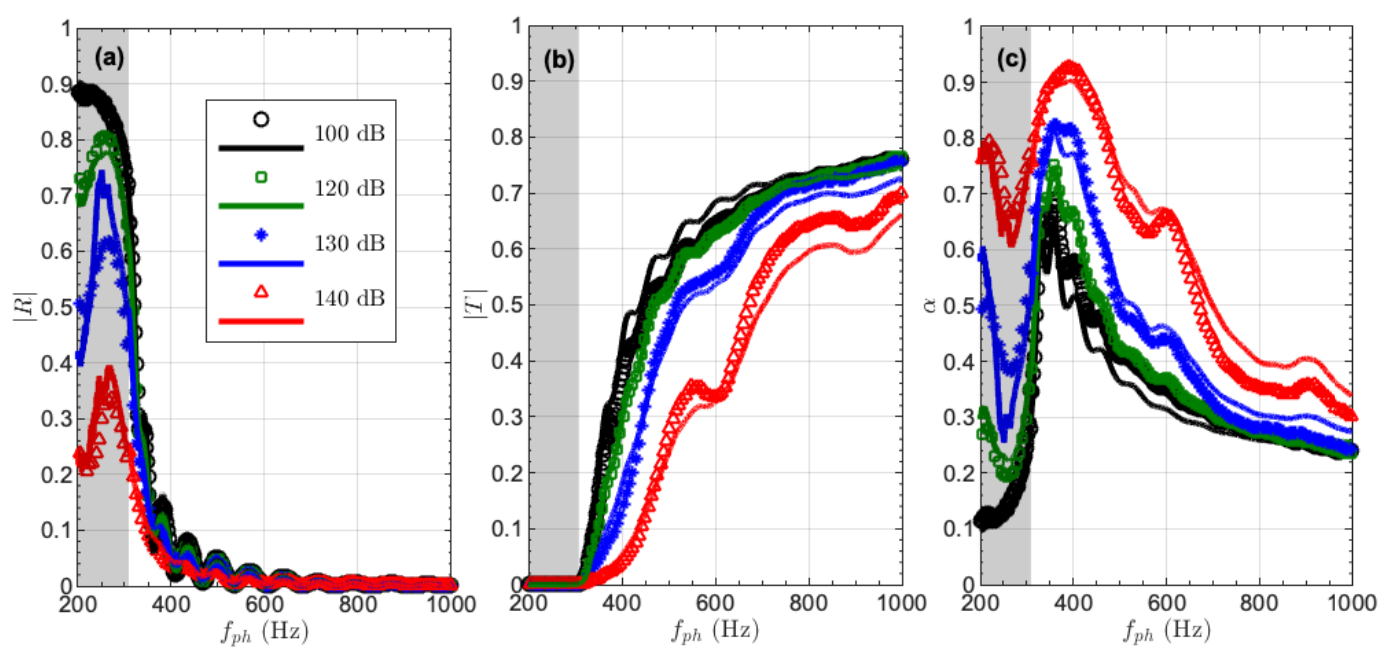

FIG. 2. (color online) Amplitude of the (a) reflection coefficients, (b) transmission coefficients and (c) absorption coefficients. Black circles (lines), green squares (lines), blue stars (lines) and red triangles (lines) present the experimental (analytical) results with a source level around $100 \mathrm{~dB}, 120 \mathrm{~dB}, 130 \mathrm{~dB}$, and $140 \mathrm{~dB}$, respectively.

the source, and we finally arrive at the following result:

$$
\begin{aligned}
p_{2 \omega_{p h}}= & \frac{2 \beta\left(-2 i \omega+\gamma_{H}\right) A_{p h}^{2}\left(2 \omega^{2}+i \gamma_{\omega} \omega\right)}{D(2 \omega, 2 k) \rho c_{0}^{2}} \\
& \times \sin \left(\frac{\Delta k_{p h}}{2} x\right) \sin \left(2 \omega_{p h} t-k_{e f f}^{p h} x\right) \\
& +A_{a}^{p h} \cos \left[2 \omega_{p h} t-k_{p h\left(2 \omega_{p h}\right)} x\right] .
\end{aligned}
$$

In Eq. (2), $\beta=1.2$ is the coefficient of nonlinearity in $\operatorname{air}^{35}, \omega=\frac{\omega_{p h} d}{\pi c_{0}}$ and $k=\frac{k_{p h} d}{\pi}$ are dimensionless form of frequency $\omega_{p h}$ and wavenumber $k_{p h}, \gamma_{H}$ and $\gamma_{\omega}$ stand for the dimensionless resistances of the side holes and the waveguide respectively, $D(\omega, k)$ is dimensionless linear dispersion relation of the system, $A_{p h}, \rho$ and $c_{0}$ are amplitude of source, density and the speed of sound respectively, $\Delta k_{p h}=k_{p h\left(2 \omega_{p h}\right)}-2 k_{p h\left(\omega_{p h}\right)}$ is the detuning parameter that describes the asynchronous secondharmonic generation, and $k_{\text {eff }}^{p h}=\frac{1}{2}\left[k_{p h\left(2 \omega_{p h}\right)}+2 k_{p h\left(\omega_{p h}\right)}\right]$ is the effective wavenumber. To account for the nonlinear losses appearing at the positions of the side holes, we add an amplitude-dependent term to the radiative losses, by using the average value of the nonlinear impedance $Z_{N L(n)}$ along the tube, and substitute into $\gamma_{H}$ (see Supplementary Material for details). An interesting feature of the solution of Eq. (2) is that it describes a field with an amplitude oscillating in space. This effect, called secondharmonic beating, is in fact a consequence of the phase mismatch, and is described by the term $\sin \left(\frac{\Delta k_{p h}}{2} x\right)$. The positions of the minima of the beating are related to the second-harmonic phase mismatch, and are given by:

$$
x_{n}=\frac{(2 n) \pi}{\Delta k_{p h}} .
$$

To illustrate the beating phenomenon and highlight the importance of nonlinear losses, we perform experiments for a specific frequency of $600 \mathrm{~Hz}$, and we measure the temporal signal at different locations uniformly distributed inside the waveguide (every $5 \mathrm{~cm}$ ). Using a Fourier analysis, we get the amplitude of the fundamental component $p_{\omega_{p h}}$ and the generated second harmonic $p_{2 \omega_{p h}}$. This experiment is repeated for three different amplitudes of the source, $A_{p h}=28 \mathrm{~Pa}$ (equiv. $120 \mathrm{~dB}$ ), $A_{p h}=89 \mathrm{~Pa}$ (equiv. $130 \mathrm{~dB}$ ) and $A_{p h}=283 \mathrm{~Pa}$ (equiv. $140 \mathrm{~dB}$ ).

The analytical and experimental results are shown in Fig. 3, where the first component amplitude is displayed in Fig. 3 (a), (c) and (e) for $120 \mathrm{~dB}, 130 \mathrm{~dB}$ and $140 \mathrm{~dB}$ respectively, and second harmonic in Fig. 3 (b), (d) and (f), for the same source amplitudes. The solid lines correspond to the analytical results, while the dashed ones present the analytical results but without taking into account the nonlinear losses. The results of the experiments are shown by the markers (circles for the first component and square for the second harmonic).

For the fundamental frequency component, we demonstrate that the influence of the nonlinear losses is not negligible when the amplitude of the source increases. For example, with a source amplitude of $140 \mathrm{~dB}$ [Fig. 3 (e)], the decrease is doubled after a propagation $3 \mathrm{~m}$ with the nonlinear losses. If only linear losses are considered (dashed line) the analytical results do not capture the experiments correctly. However, using our additional nonlinear impedance we obtain the solid line which is in a very good agreement with the experiments.

The beating of the second harmonic is clearly shown in the panels (b), (d) and (f) of Fig. 3. Once again, we demonstrate the strong influence of the nonlinear losses on the second-harmonic generation and the resulting beating. The difference between the results including and neglecting nonlinear losses is increasing with the amplitude of the wave, illustrating the amplitude dependence of this phenomenon. Without the nonlinear losses, 

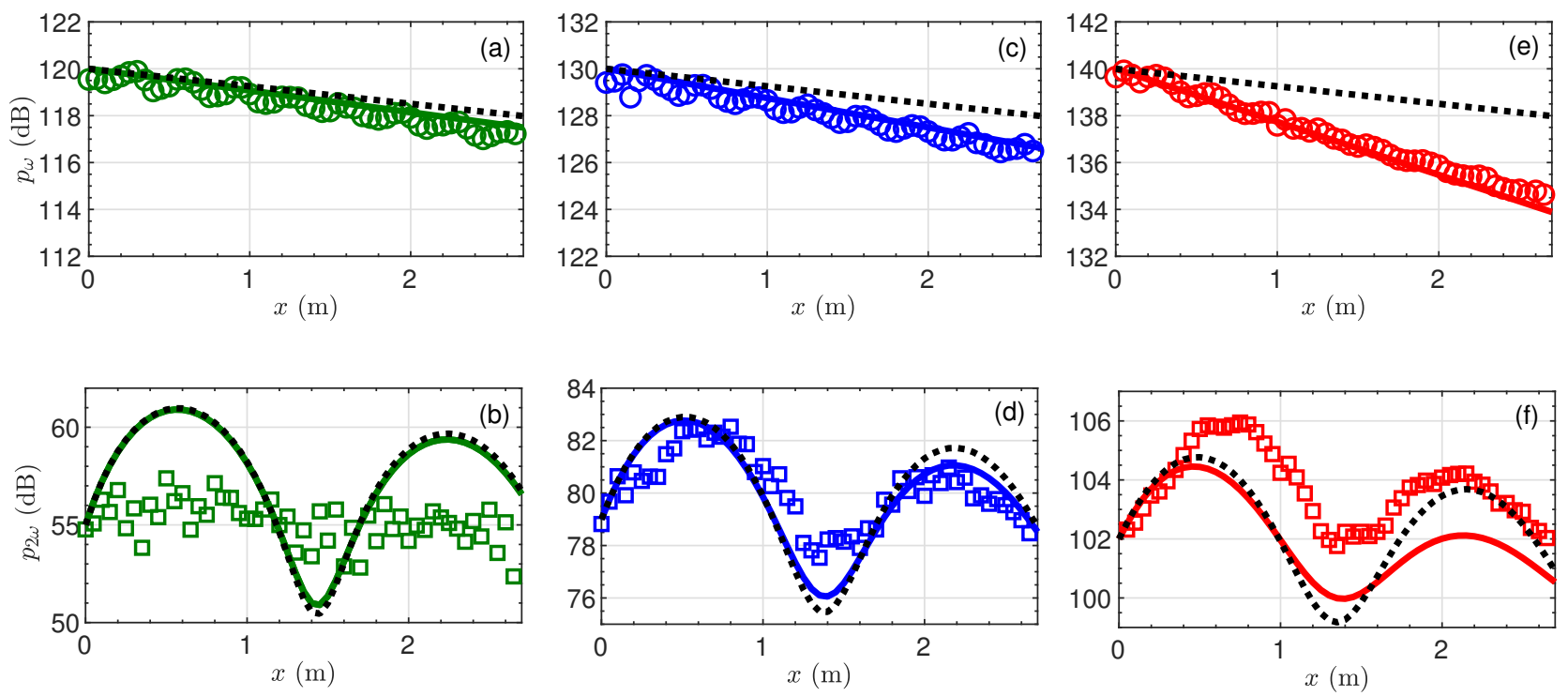

FIG. 3. (color online) Experimental and analytical results for the second-harmonic generation. The source is of the form $A_{p h} \sin \left(\omega_{p h} t\right)$ amplitude, $120 \mathrm{~dB}(\mathrm{a})-(\mathrm{b}), 130 \mathrm{~dB}(\mathrm{c})-(\mathrm{d})$, and $140 \mathrm{~dB}(\mathrm{e})-(\mathrm{f})$. The symbols (solid lines) are experimental (analytical) results. Circles (squares) stand for the experimental results of $p_{\omega_{p h}}\left(p_{2 \omega_{p h}}\right)$. Dotted lines are analytical results without considering nonlinear losses.

the amplitude of the beating during propagation is much less decreasing. These results are confirmed by the experiments, and our analytical approximations capture the observed behavior quite well, apart from the case shown in Fig. 3 (b) and (f). In Fig. 3 (b), the experimental result (green squares) does not show the beatings of $p_{2 \omega}$ in space, because the excitation amplitude $(120 \mathrm{~dB})$ is not strong enough, and the generated second harmonic is hidden in the experimental noise. In Fig. 3 (f), the experimental result (red squares) shows the significant decrease of the amplitude of the beatings, which could validate our prediction about the importance of considering nonlinear losses. However, there is still $2 \mathrm{~dB}$ difference between analytical and experimental results, probably because our analytical approach only considers the second-harmonic generation, while the third harmonic exists in this case, due to large amplitude of the wave excitation (140 dB). Finally, as predicted by the model [see Eq. (3)], the position of the minima of the second harmonic, due to the beating, are not sensible to the source amplitude, and are very well captured by the analytical model.

In conclusion, we have studied both experimentally and analytically nonlinear wave scattering in an 1D airfilled waveguide periodically side-loaded by holes. We have treated nonlinear losses using an analytical model based in transfer matrix theory. By performing scattering experiments with different amplitudes we have determined the amplitude-dependent reflection, transmission and absorption coefficients of the structure, and our analytical results where found to capture the observed enhanced absorption. Additionally, we have performed experiments in order to observe the beating phenomenon of the generated second harmonic. The importance of nonlinear losses was also illustrated in this case, and our analytical model captures the experimental results quite accurately. Our results concerning nonlinear losses, especially regarding the observed large value of the absorption coefficient in a relatively large bandwidth, are encouraging for the design of amplitude-dependent absorbers. On the other hand, the emergence and the good analytical description of the second-harmonic generation could be used for controlling and tailoring nonlinear waves. Finally, it would be interesting to study harmonic generation in higher dimensional acoustic metamaterials.

\section{SUPPLEMENTARY MATERIAL}

See the Supplementary Material for details on the transfer matrix theory considering the experssions for the nonlinear part of the impedances of the holes, as well as for the transmission line approach used in this work to analyze the beating phenomenon.

\section{DATA AVAILABILITY STATEMENT}

The data that support the findings of this study are available from the corresponding author upon reasonable request. 
${ }^{1}$ S. Cummer and D. Schurig, "One path to acoustic cloaking," New J. Phys. 9 (2007).

${ }^{2}$ D. Torrent and J. Sanchez-Dehesa, "Acoustic cloaking in two dimensions: a feasible approach," New J. Phys. 10 (2008).

${ }^{3}$ B. I. Popa, L. Zigoneanu, and S. A. Cummer, "Experimental acoustic ground cloak in air," Phys. Rev. Lett. 106(25) (2011).

${ }^{4}$ C. Faure, O. Richoux, S. Félix, and V. Pagneux, "Experiments on metasurface carpet cloaking for audible acoustics," Appl. Phys. Lett. 108 (2016)

${ }^{5}$ G. Ma, M. Yang, S. Xiao, Z. Yang, and P. Sheng, "Acoustic metasurface with hybrid resonances," Nat. Mater. 13, 873-878 (2014).

${ }^{6}$ V. Romero-García, G. Theocharis, O. Richoux, A. Merkel, V. Tournat, and V. Pagneux, "Perfect and broadband acoustic absorption by critically coupled sub-wavelength resonators," Sci. Rep. 6 (2016).

${ }^{7}$ A. Climente, D. Torrent, and J. Sanchez-Dehesa, "Sound focusing by gradient index sonic lenses," Appl. Phys. Lett. 97 (2010).

${ }^{8}$ J. Welter, S. Sathish, D. Christensen, P. Brodrick, and M. Cherry, "Focusing of longitudinal ultrasonic waves in air with an aperiodic flat lens." J. Acoust. Soc. Am. 130, 2789-2796 (2011).

${ }^{9} \mathrm{~J}$. Zhao, B. Bonello, and O. Boyko, "Focusing of the lowestorder antisymmetric lamb mode behind a gradient-index acoustic metalens with local resonators," Phys. Rev. B 93 (2016).

${ }^{10}$ X. Ni, C. He, X.-C. Sun, X. ping Liu, M.-H. Lu, L. Feng, and Y.-F. Chen, "Topologically protected one-way edge mode in networks of acoustic resonators with circulating air flow," New J. Phys. 17 (2015).

${ }^{11}$ V. Peano, C. Brendel, M. Schmidt, and F. Marquardt, "Topological phases of sound and light," Phys. Rev. X 5 (2015).

${ }^{12}$ Y.-G. Peng, C.-Z. Qin, D.-G. Zhao, Y.-X. Shen, X.-Y. Xu, M. Bao, H. Jia, and X.-F. Zhu, "Experimental demonstration of anomalous floquet topological insulator for sound," Nature Commun. 7 (2016).

${ }^{13}$ B. Liang, B. Yuan, and J. C. Cheng, "Acoustic diode: rectification of acoustic energy flux in one-dimensional systems," Phys. Rev. Lett. 103 (2009).

${ }^{14}$ X.-F. Li, X. Ni, L. Feng, M.-H. Lu, C. He, and Y.-F. Chen, "Tunable unidirectional sound propagation through a soniccrystalbased acoustic diode," Phys. Rev. Lett. 106 (2011).

${ }^{15}$ N. Boechler, G. Theocharis, and C. Daraio, "Bifurcation-based acoustic switching and rectification," Nature Mater. 10, 665 (2011)

${ }^{16}$ C. M. Donahue, P. W. J. Anzel, L. Bonanomi, T. A. Keller, and C. Daraio, "Experimental realization of a nonlinear acoustic lens with a tunable focus," Appl. Phys. Lett. 104, 014103 (2014).

${ }^{17}$ V. Achilleos, O. Richoux, G. Theocharis, and D. Frantzeskakis, "Acoustic solitons in waveguides with helmholtz resonators: Transmission line approach," Phys. Rev. E 91 (2015).

${ }^{18}$ V. C. Henríquez, V. M. Garcia-Chocano, and J. Sánchez-Dehesa, "Viscothermal losses in double-negative acoustic metamaterials,"
Phys. Rev. Applied. 8 (2017).

${ }^{19}$ R. Graciá-Salgado, V. García-Chocano, D. Torrent, and J. Sánchez-Dehesa, "Negative mass density and density-near-zero quasi-two-dimensional metamaterial: Design and applications," Phys. Rev. B 88 (2013).

${ }^{20} \mathrm{C}$. E. Bradley, "Time-harmonic acoustic bloch wave propagation in periodic waveguides. part iii. nonlinear effects," J. Acoust. Soc. Am. 98, 2735 (1995).

${ }^{21}$ J. Zhang, V. Romero-García, G. Theocharis, O. Richoux, V. Achilleos, and D. J. Frantzeskakis, "Second-harmonic generation in acoustic waveguides loaded with an array of side holes," Acta Acust united Ac. 104, 235-242 (2018).

${ }^{22} \mathrm{~J}$. Zhang, V. Romero-García, G. Theocharis, O. Richoux, V. Achilleos, and D. J. Frantzeskakis, "Dark solitons in acoustic transmission line metamaterials," Applied Science 8(7) (2018).

${ }^{23}$ M. Atig, J. Dalmont, and J. Gilbert, "Termination impedance of open-ended cylindrical tubes at high sound pressure level." Comptes Rendus Mécanique 332(4), 299-304 (2004).

${ }^{24}$ J. M. Buick, A. M., D. Skulina, D. Campbell, J.P.Dalmont, and J. Gilbert, "Investigation of non-linear acoustic losses at the open end of a tube." J. Acoust. Soc. Am. 129(3), 1261-72 (2011).

${ }^{25}$ O. Lombard, C. Barrière, and V. Leroy, "Nonlinear multiple scattering of acoustic waves by a layer of bubbles," EPL 112 (2015).

${ }^{26}$ L. Fan, H. Ge, S. yi Zhang, H. fei Gao, Y. hui Liu, and H. Zhang, "Nonlinear acoustic fields in acoustic metamaterial based on a cylindrical pipe with periodically arranged side holes," J. Acoust. Soc. Am 133, 3846 (2013).

${ }^{27}$ J. P. Dalmont, J. Kergomard, and X. Meynial, C.R. Acad. Sci. II, 309 (1989).

${ }^{28}$ G. Theocharis, a. V. R.-G. O. Richoux, and V. Tournat, "Limits of slow sound propagation and transparency in lossy, locally resonant periodic structures," New J. Phys. 16 (2014).

${ }^{29}$ B. H. Song and J. S. Bolton, "A transfer-matrix approach for estimating the characteristic impedance and wave numbers of limp and rigid porous materials," J. Acoust. Soc. Am. 107, 1131 (2000).

${ }^{30}$ S. Lee, C. Park, Y. Seo, Z. Wang, and C. Kim, "Composite acoustic medium with simultaneously negative density and modulus," Phys. Rev. Lett. 104 (2010).

${ }^{31}$ C. Zwikker and C.W.Kosten, Sound absorbing materials (Elsevier, New York, 1949).

${ }^{32}$ M. A. Temiz, J. Tournadre, I. L. Arteaga, and A. Hirschberg, "Non-linear acoustic transfer impedance of micro-perforated plates with circular orifices," J. Sound Vib. 366, 418-428 (2016).

${ }^{33}$ J. Disselhorst and L. V. Wijngaarden, "Flow in the exit of open pipes during acoustic resonance." J. Fluid Mech. 99(2), 293-319 (1980).

${ }^{34}$ M. Peters and A. Hirschberg, "Acoustically induced periodic vortex shedding at sharp edged open channel ends: simple vortex models." J. Sound Vib. 161(2), 281-299 (1993).

${ }^{35}$ M. Hamilton and D. T. Blackstock, Nonlinear Acoustics (Academic Press, San Diego, CA, 1998). 